\title{
Los caminos rurales desde una perspectiva histórica: antecedentes y novedades del Plan de Caminos de Fomento Agrícola (Argentina, 1956)
}

As estradas rurais de uma perspectiva histórica: história e evolução do Plan de Caminos de Fomento Agrícola (Argentina, 1956)

The rural roads from a historical perspective: history and changes of Plan de Caminos de Fomento Agrícola (Argentina, 1956)

Alejandra Laura Salomón ${ }^{*}$

\section{Resumen}

Los caminos rurales cumplen un rol importante en el desarrollo de las actividades productivas y en la mejora de la condiciones de vida de la población rural. Debido a su impacto socio-económico y a que resulta un área relativamente poco estudiada, el propósito del trabajo es reconstruir las políticas públicas sobre los caminos rurales argentinos, con el foco puesto en el Plan de Caminos de Fomento Agrícola (sancionado en 1956). Se analizarán sus antecedentes y novedades en un contexto signado por la modernización del agro pampeano y la centralidad de la idea de desarrollo. Asimismo, se examinarán las derivaciones normativas e institucionales en el gobierno de la provincia de Buenos Aires.

Palabras clave: Argentina. Caminos. Rural.

\section{Introducción}

En un interesante trabajo Theda Skocpol señala que los significados de la vida pública y las formas colectivas a través de las cuales los grupos adquieren conciencia de los objetivos políticos y trabajan para alcanzarlos surgen de los puntos de encuentro de los Estados y las sociedades (SKOCPOL, [1989] 2007, p. 193). Sus dichos incitan a reflexionar sobre la potencialidad que detentan las políticas públicas cuando son el fruto

Doctora en Ciencias Sociales y Humanas por la Universidad Nacional de Quilmes (UNQ), Argentina. Profesora de grado y posgrado de la UNQ.

E-mail: alejandralaurasalomon@gmail.com

Recebido em: 18/08/2017 - Aprovado em: 20/10/2017 http://dx.doi.org/10.5335/hdtv.18n.2.8075 
de la combinación de iniciativas estatales y vecinales. A este objetivo apuntó el Plan de Caminos de Fomento Agrícola (sancionado en 1956), el cual puede ser considerado punto de inflexión dentro de las obras públicas en materia vial rural.

Los caminos rurales - aquellos que permiten el acceso a las explotaciones agropecuarias y que corresponden a las redes viales secundaria o terciaria, bajo control provincial o municipal - cumplen un importante rol en el desarrollo de las actividades productivas y en la mejora de la condiciones de vida de la población rural. No sólo constituyen elementos indispensables para la producción y comercialización de la producción, sino también para la comunicación, la educación y la salud. En un país vasto y con una infraestructura de transporte insuficiente, dicho Plan puede leerse como la actualización de un anhelo decimonónico, en pos de la integración.

En la Argentina, no abundan los trabajos sobre vialidad, y la mayor parte de ellos se dedican a la primera mitad del siglo XX. Se la ha abordado desde una perspectiva económica, en relación a los avances de la industria automotriz (GARCÍA HERAS, 1985), la incidencia de los importadores de automóviles (OSPITAL, 2002) y su complementariedad con el ferrocarril (GÓMEZ; TCHORDONKIAN, 2014). Con una impronta social, algunos estudios se han abocado a la vinculación entre automóvil, camino y turismo (SCARZANELLA, 1998; OSPITAL, 2005) y las intervenciones públicas en materia de vialidad y turismo del Automóvil Club Argentino y del Touring Club Argentino (PIGLIA, 2014). También ha sido exami- nada la contribución de las carreras automovilísticas y del turismo a la construcción de un imaginario del camino y a la difusión del automóvil (BALLENT; GORELIK, 2002; BALLENT, 2005). Por otra parte, pueden registrarse aportes desde la historia del Estado, por ejemplo, sobre la acción de los ingenieros en las reparticiones estatales (BALLENT, 2008) y la relación entre expertos y saberes viales de Estados Unidos y la Argentina (GRUSCHETSKY, 2012). En tanto, es muy poco lo escrito sobre la provincia de Buenos Aires (PIGLIA, 2011; FERNÁNDEZ, 2016).

En vista de su impacto socio-económico y de que resulta aún un área poco estudiada, el propósito del trabajo es reconstruir las políticas públicas sobre los caminos rurales, con el foco puesto en el Plan de Caminos de Fomento Agrícola. Se analizarán sus antecedentes y novedades en un contexto signado por la modernización del agro y la centralidad de la idea de desarrollo, así como se examinarán las derivaciones normativas e institucionales en la provincia de Buenos Aires. En base a fuentes oficiales y publicaciones periódicas, se atenderán dos interrogantes: ¿Qué novedades introdujo el Plan de Caminos de Fomento Agrícola dentro de las políticas viales? ¿De qué modo se articularon los diferentes niveles de gobierno (nacional, provincial y municipal) y la sociedad civil en materia de planificación, financiación, ejecución y mantenimiento de la red vial en el agro?

Para este trabajo resulta un aporte la perspectiva de Oszlak y O’Donnell (1981), para quienes las políticas estatales constituyen un conjunto de acciones y omisiones que manifiestan una determinada modali- 
dad de intervención del Estado en relación con una cuestión que concita atención, interés o movilización de otros actores en la sociedad civil. Es decir, interactúan con un proceso social tejido alrededor de temas que las originan y suponen una priorización de los mismos. La selección prioritaria compone la agenda pública, que nos muestra cuál es la percepción de los poderes públicos sobre lo que se debe atender y resolver (TAMAYO SÁEZ, 1997).

\section{Los caminos municipales a mediados del siglo XX}

Desde el punto de vista técnico, la clasificación que históricamente se ha hecho de los caminos que componen la red total del país resulta de dividirla en tres sistemas: nacional, provincial y municipal. Tal enfoque es el que inspiró en la Ley Nacional de Vialidad (1932) la definición de las redes, las que a su vez variaban de acuerdo su longitud e intensidad de circulación. La red municipal, también denominada indistintamente vecinal, comunal o terciaria, era la más extensa de las tres y se la establecía por exclusión (no incluida en las demás).

En la provincia de Buenos Aires, a mediados de la década de 1950 los datos estadísticos indican que, sobre $109.169 \mathrm{~km}$ de caminos en total, los de jurisdicción municipal ascendían estimativamente a $65.000 \mathrm{~km}$, es decir, representaban el 59,54\%. La red nacional estaba pavimentada en un $57,7 \%$, la red provincial en un $5 \%$ y la municipal prácticamente no contaba con pavimento. ${ }^{1} \mathrm{Al}$ igual que en resto del país y tal como cuestionaba la prensa local, casi todos los caminos comu- nales eran de tierra y precarios, y por ellos el tránsito era condicional y de baja intensidad. ${ }^{2}$ La falta de conservación permanente los hacía proclives a la erosión y al anegamiento. De acuerdo al director de la Dirección Nacional de Vialidad (DNV), el equipo de las comunas bonaerenses para la conservación podía estimarse entre el 10 y el 15\% de las necesidades normales. ${ }^{3}$ Otro punto para destacar es la carencia de un inventario de la red municipal, cuya evolución, en permanente movilidad, respondía más a la subdivisión de la propiedad inmobiliaria antes que a la planificación.

La primera política respecto a los caminos comunales data de 1907: la ley 5.315, denominada Ley Mitre, que fomentó hasta 1947 los caminos de acceso a las estaciones ferroviarias. Establecía que el 3\% del producido líquido por las compañías ferroviarias sería aplicado a la construcción o mantenimiento de los puentes y caminos de los municipios o departamentos cruzados por la línea, en primer término de los caminos que condujeran a las estaciones y en proporción a la extensión de vías en cada provincia. ${ }^{4}$ De modo que la planificación y edificación de caminos, complementarios al ferrocarril, fueron realizadas con los aportes y el criterio de las compañías ferroviarias. No existía un plan estatal que coordinara las reparticiones nacionales, provinciales y municipales y que atendiera los gastos de conservación permanente. ${ }^{5}$ Para paliar este problema, en 1935 se responsabilizó a las provincias de la tarea, pero la falta de recursos y de maquinarias imposibilitó el cumplimiento de la norma. Además, como los aportes viales se supeditaban a los productos líquidos, se ge- 
neró una gran desigualdad entre las provincias. ${ }^{6}$ Con el paso de los años, y en particular a partir de los años treinta, las compañías ferroviarias perdieron injerencia en el sector caminero, paralelamente a su declinación y a la competencia de los automotores. Entre 1933 y 1943, en promedio, el 20\% del tendido vial fue el resultado de la aplicación de la ley 5.315; en el resto participó el Estado nacional, ya sea a través de la coparticipación nación-provincias o directamente con obras federales (GÓMEZ; TCHORDONKIAN, 2014, p. 19). Mientras se reducía la contribución empresarial, el desarrollo de los caminos municipales quedó librado al esfuerzo del vecindario, de las comisiones de fomento y de los intendentes.

En la década de 1930, al tiempo que la sustitución de importaciones emergía como una alternativa para paliar los inconvenientes derivados del cierre de mercados exteriores, la necesidad de "vivir con lo propio" impulsó un acercamiento con el interior del país y motorizó iniciativas vinculadas con el desarrollo de los caminos (BALLENT; GORELIK, 2002). La principal tracción a la vialidad tuvo lugar a través de la ley 11.658/32, que creó un fondo nacional para el estudio, trazado, construcción, mejoramiento y conservación de caminos y obras anexas, obtenido por un impuesto sobre la nafta y los lubricantes. Conjuntamente, la creación de la DNV - dentro del Ministerio de Obras Públicas (MOP) - fue pensada para proyectar y ejecutar la red nacional de caminos y así dinamizar las actividades económicas y lograr la efectiva integración del territorio (PIGLIA, 2014). Dotada de autarquía, la presencia en su directorio de asociaciones agra- rias, entidades automovilísticas y empresas del transporte la revestía de un aura de eficiencia. El proyecto original del director de DNV, el ingeniero Justiniano Allende Posse, replicaba el modelo norteamericano en pos de la expansión de carreteras para servir a la producción, aunque la creciente importancia del turismo modificó parcialmente tal proyecto primigenio (OSPITAL, 2005). La ley fijó también una "ayuda federal" destinada a las noveles direcciones provinciales de vialidad. Junto a ellas, el intenso accionar de la DNV se tradujo en el tendido de una red nacional o troncal de carreteras, y de los caminos provinciales que empalmaban con ella. Los caminos terciarios no se habían convertido, por entonces, en una preocupación estatal inmediata. Ello obedece a que el criterio de la cantidad de tránsito y del bajo costo, en un conflictivo contexto económico, determinaba las prioridades camineras.

En materia de vialidad, el ascenso de Juan D. Perón a la presidencia en 1946 significó una continuidad (GÓMEZ; TCHORDONKIAN, 2014, p. 22), exceptuando la pérdida de autarquía de la DNV, que fue reorganizada y transformada en Administración General de Vialidad Nacional. Las políticas gubernamentales siguieron priorizando la explotación de recursos productivos y turísticos y la integración nacional. En segunda instancia, se bregó por la conectividad con países limítrofes para mejorar la defensa y la exportación de productos. Cabe destacar la promoción de autopistas como forma de reducir accidentes, optimizar el consumo de automotores, descentralizar zonas urbanizadas, incrementar el rendimiento de las calzadas, fomentar la higie- 
ne y mejorar la defensa nacional. La visión sistemática del territorio y la preeminencia del criterio económico hicieron que se optara por la construcción de una extensa red de caminos nacionales de bajo costo. Sin embargo, el encarecimiento de la mano de obra y los insumos, junto a mayores gastos en conservación, actuó en detrimento del aprovechamiento de los fondos disponibles, pese a haberse incrementado.

En suma, no se había configurado una política orgánica y permanente. Las redes municipales, generalmente de tierra, por mucho tiempo no gozaron de la atención política de las autoridades, ni consideración en la opinión pública a nivel nacional. Lo expresó con claridad el diputado Oscar Alende en 1954 "ni los diarios ni las revistas de difusión popular dicen una sola palabra sobre la gravedad del problema vial $[\ldots]^{\prime \prime}{ }^{7}$ Tendía a ser la prensa local la que, recogiendo el estado de ánimo de los productores, destacaba la insuficiencia y precariedad de vías de comunicación en el agro. También algunos ingenieros insistían en la mayor injerencia estatal en eventos del campo disciplinar.

Pese a ser un reclamo que de modo marginal ciertos actores reiteraban sobre todo desde la década de 1930, la caída del peronismo en 1955 fue la circunstancia que, desde el punto de vista de la política gobernante, mostró oportuna la propuesta. El crecimiento del transporte automotriz, la paulatina pérdida de la carga del ferrocarril y la expansión agraria plantearon la necesidad de dar organicidad al desarrollo de los caminos alimentadores de la red troncal. En este marco, el Plan de Caminos de Fomento Agrícola fue la cristalización de una nueva concepción sobre el tema, al establecer una serie de principios básicos sobre los cuales debían ser tendidos los caminos. Si bien no fue nueva la asociación de éstos a la producción agraria, sí lo fue el modo el modo en que se manifestó la intervención del Estado en la materia.

\section{Modernización del agro, desarrollo y transportes}

Modernización y el desarrollo fueron prioridades en la agenda estatal tras el derrocamiento de Juan D. Perón. En materia económica, una de las principales consignas de la Revolución Libertadora consistía en la racionalidad y la planificación, en contraste con la demagogia y la dilapidación de recursos de la "segunda tiranía" peronista. Los informes económicos de Raúl Prebisch, prestigioso presidente de la CEPAL y asesor gubernamental, incentivaron un rico debate intelectual acerca del rol del capital extranjero y del Estado, así como de la relación entre el agro y la industria para el desarrollo económico (ALTAMIRANO, 1998). Para el asesor, la postración de la producción agraria constituía un serio problema, por lo que aconsejaba el aumento de la productividad a partir de la mecanización, nuevas formas de producción, exportaciones e investigación científica. ${ }^{8} \mathrm{Su}$ estrategia de desarrollo contenía políticas sin demora para el agro, entre ellas, fijar precios favorables, construir silos, facilitar la importación de maquinaria y crear un instituto de investigación y difusión tecnológica. Asimismo propiciaba la solución de carencias que desalentaban 
la modernización de la empresa rural: los transportes, viales y ferroviarios.

En un país convulsionado políticamente difícilmente podía llevarse adelante un ambicioso programa de medio y largo plazo, por lo que la conducción económica estuvo a la defensiva (GERCHUNOFF; LLACH, 2010, p. 240; FERRER; ROUGIER, 2012, p. 298). De todos modos, se adoptaron políticas más favorables para el sector agropecuario: una fuerte devaluación, la disolución del IAPI, la liberación del precio de la carne, la fijación de precio para los cereales, beneficios impositivos, créditos subsidiados y la derogación del congelamiento de los arrendamientos. La recomposición de incentivos al agro, tendencia que ya se vislumbraba desde finales del gobierno peronista, facilitó la obtención de recursos. Además, influyó en la conducta de los productores el cambio en la oferta tecnológica, dando lugar a un proceso de expansión agrícola, inicialmente lenta (BARSKY; GELMAN, 2009, p. 333). Otra de las medidas relevantes del período fue la creación del Instituto Nacional de Tecnología Agropecuaria (INTA), institución destinada a la tecnificación y al mejoramiento de la empresa agraria y de la vida rural, en tiempos en que la cuestión tecnológica comenzó a ser problematizada como la llave maestra para salir del estancamiento. Este paradigma abrevaba en las ideas desarrollistas que promovían el rol decisivo del Estado para modernizar el aparto productivo.

El análisis del período nos muestra que la decisión con la que se encaró la cuestión de los caminos rurales no se debió solamente a un diagnóstico de la crisis, sino también a un proyecto de desarrollo nacional de un sector insatisfecho hasta entonces con el gobierno nacional. Vialidad y desarrollo constituían dos caras de la misma moneda: "Visto que es propósito del Gobierno Provisional impulsar las obras de vialidad en todo el territorio del país y en la medida que su desarrollo económico exige" ${ }^{\prime \prime}$

El gobierno depuesto era denostado por sus opositores como ineficiente, demagogo, centralizador y autoritario. La oposición a esta imagen guió numerosos decisiones del gobierno provisional. Al igual que en otras reparticiones, en el caso de la vial bregó por montar un aparato moderno, administrador y eficiente, diferente del heredado. En base a esta premisa, la repartición desempeñó un papel importante en el ensayo de políticas viales y marcó algunas discontinuidades respecto al gobierno peronista. Primero, el decreto-ley 22.297/56 le restituyó la autarquía administrativa y el nombre de DNV. El ingeniero Justiniano Allende Posse volvió a ser nombrado director de esta última, y con él retornaron otros ingenieros que acreditaban experiencia pública en los años treinta. Allende Posse era un acérrimo defensor de un sistema federal basado en dos principios: acción subsidiaria (implica que no hace un organismo mayor lo que pueden hacer mejor organismos más pequeños) y totalidad (convergencia de objetivos en un plan común). ${ }^{10}$ Segundo, el decretoley 23.033/56 la dotó de mayores recursos y créditos y el decreto-ley 3.103/56 determinó el aumento del aporte empresarial al fondo de vialidad..$^{11}$ Tercero, el decreto-ley 505/58 le devolvió la completa autarquía a la DNV y los fondos propios, y creó el Consejo Vial Federa para imprimir celeridad a las obras. 
Además, estableció modificaciones en la estructura financiera, al contemplar no sólo la ampliación de recursos sino una nueva distribución de los mismos en beneficio de las provincias. $^{12}$

Las medidas fueron aplicadas dentro de un contexto político-ideológico que defendía la descentralización y la iniciativa privada como pilares del desarrollo económico. Por entonces las Naciones Unidas divulgaron la expresión desarrollo de la comunidad para designar aquellos procesos que combinaban esfuerzos de la población y el gobierno para mejorar el nivel de vida de las comunidades. ${ }^{13}$ En este escenario, no fue casual que en 1961 se reuniera el Primer Congreso Vial Municipal de la Provincia de Buenos Aires, abocado a una reflexión explícita sobre el rol del municipio y de los vecinos en materia de vialidad, contribuyendo a la concepción de ésta como un problema también local. En el II Congreso Vial Municipal, celebrado en 1965, el presidente de la Comisión Organizadora, Bernardo Calderwood, enfatizó el imperativo de la "descentralización vial", así como de la "cooperación vial para el progreso tanto en el orden social como económico y político con la mira de un mejor bienestar general" ${ }^{14}$ Entre otras cuestiones, se discutió sobre el papel de los consorcios y las comisiones municipales y se propuso la creación de un fondo vial municipal para obras viales rurales.

Otros hechos dan cuenta de la revitalización de la cuestión vial. En 1957 se erigió la Escuela de Ingeniería de Caminos, dependiente de la Dirección de Vialidad de la Provincia de Buenos Aires, que funcionó cómo ámbito de transmisión universitaria.
Comenzó a publicarse en 1954 Noticias Camineras y al año siguiente la revista Carreteras, órganos de prensa de la recientemente creada Asociación Argentina de Carreteras. Desde allí y desde el VI Congreso Panamericano de Carreteras (1954) se pugnaba por los caminos vecinales, tomando como modelo las experiencias extranjeras (Venezuela, México, Perú y EEUU).${ }^{15}$ De modo que, pese a que desde los años veinte se organizaban congresos y exposiciones sobre vialidad, fue a partir de los años cincuenta cuando se hizo más visible la dupla vialidad/municipalidad.

\section{El Plan de Caminos de Fomento Agricola}

El Plan de Caminos de Fomento Agrícola definió, por primera vez, una política explicita de desarrollo de caminos rurales a través del decreto-ley 9.875/56, , $^{16}$ "para beneficio de todo el país y en modo particular, para beneficio directo e inmediato de los productores rurales" ${ }^{17}$ Entre los fundamentos del proyecto se declaraba la necesidad de restaurar la economía agraria a través de un rápido y económico desplazamiento de las cosechas, lo cual tendería a la disminución de costos, al aumento del área sembrada y al arraigo de la población campesina. El objetivo puntual era la construcción, mejoramiento y conservación de caminos que comunicaran las unidades o centros de producción agrícola con las estaciones ferroviarias, puertos y caminos pavimentados. Dado que estos caminos se emplazarían en jurisdicción provincial o municipal, se necesitaba la conformidad de las autoridades subnacionales. 
A los efectos de ese decreto-ley, se instituyó un fondo de m\$n 1.500.000, a invertirse en cinco años. Se crearon dos sistemas de caminos agrícolas, denominados de primera y segunda categoría. Los de primera categoría (fondo A), delegados a las provincias acogidas al plan, constituirían colectoras con conexión con las redes pavimentadas provinciales o nacionales. Los de segunda categoría (fondo B) estarían formados por los caminos de penetración directa y facilitarían la salida de la producción agrícola desde las chacras hasta las redes de primera categoría o redes provinciales o nacionales pavimentadas o bien los centros de consumo, distribución y transporte. Estos últimos caminos se realizarían por el régimen de consorcios camineros y la administración de los fondos quedaría reservada a Vialidad Nacional.

El plan preveía un esquema de financiamiento y una estructura organizativa descentralizada respecto a los caminos rurales. Aquellas municipalidades que adscribieran a la normativa deberían crear dos organismos. Por un lado, una Comisión Vial honoraria de cinco miembros presidida por el intendente que se abocaría al estudio de las necesidades viales locales, así como al impulso, la aprobación, la administración y el contralor de los consorcios. Por otro, los consorcios camineros, constituidos por al menos cinco vecinos interesados en la construcción de un camino. Los mismos tendrían a su cargo la ejecución y financiación del aporte mínimo del $20 \%$ del costo de los trabajos. Podrían ejecutar las obras mediante licitación, concurso de precios, adjudicación directa o bien por administración; y su aporte se plasmaría con dinero, servicios o materiales.
Vale aclarar que si bien ya detentaban status legal desde los años treinta, fue enaltecida su función y precisado su funcionamiento.

He aquí entonces la fijación de dos principios básicos: la intervención de la municipalidad y el aporte del beneficiario directo. Considerada como sostén fundamental para el desarrollo de los caminos vecinales, los poderes centrales le brindaban asistencia técnica y económica a la municipalidad, a cambio de su participación en las decisiones y capacidad de acción. Otro aspecto importante de la ley se refiere a la contribución de beneficiarios directos del camino, sea en mano de obra, dinero, equipos, cesiones de tierra, etc. En este esquema, los intereses privados, al tomar la iniciativa, eran incorporados al esquema estatal. ${ }^{18}$ Se procuraba así posicionarlos como legítimos interlocutores del Estado en representación de un interés local o sectorial. Dicha incorporación podría contemplarse de un modo más amplio, como garantía de despolitización de las instituciones públicas. En tal sentido, recordemos que en los discursos de los gobernantes posteriores a 1955, la democratización y moralización de la política se convirtió en una de las principales banderas retóricas. De modo que el Plan, al fortalecer los consorcios y las comisiones municipales como instancias de acción pública, pautó una nueva delimitación de espacios y funciones entre Estado y sociedad civil. Para ello el gobierno dictó el Reglamento de los Consorcios Camineros Vecinales y los Estatutos de las Comisiones Viales. ${ }^{19}$ Además, lo dio a conocer en el ámbito rural, para lo cual colaboró la Asociación Argentina de Carreteras con la impresión de afiches y folletos para su difusión. ${ }^{20}$ 
Contó con el respaldo de la prensa la que, como constructora de la realidad pública, se hizo eco del lanzamiento de la medida destacando su importancia económica. ${ }^{21}$

Inicialmente, el Plan previó, como único medio de financiamiento, los aportes del gobierno nacional, pero luego fueron incorporados fondos provenientes de las retenciones a las exportaciones, otorgándole una estructura permanente. Los resultados obtenidos son elocuentes al dar cuenta de una significativa inversión en la red vial.
En cuanto al sistema de caminos de primera categoría, hacia 1963 la longitud construida fue de $10.000 \mathrm{~km}$ y la inversión realizada, de m\$n 500.000.000. ${ }^{22}$ El sistema de caminos de segunda categoría contó con longitud construida y/o conservada de $70.000 \mathrm{~km}$ y una inversión de m\$n 1.383.167.148 (Cuadro 1). Estas cifras son presentadas como indicadores del vigor del Plan, aunque exigen de estudios diacrónicos más precisos que exceden los límites de este trabajo.

Cuadro 1 - Síntesis de la labor realizada desde la iniciación del Plan de Caminos de Fomento Agrícola hasta el 15/4/1963 (fondo B)

\begin{tabular}{|c|c|c|c|c|c|c|c|}
\hline \multirow{2}{*}{ Provincias } & \multirow{2}{*}{$\begin{array}{c}\text { Comisiones } \\
\text { Viales }\end{array}$} & \multirow{2}{*}{$\begin{array}{l}\text { Consorcios } \\
\text { Camineros }\end{array}$} & \multirow{2}{*}{$\begin{array}{c}\mathrm{N}^{0} \\
\text { obras }\end{array}$} & \multirow{2}{*}{ Longitud (km) } & \multirow{2}{*}{$\begin{array}{l}\text { Presupuesto } \\
(\mathrm{m} \$ \mathrm{n})\end{array}$} & \multicolumn{2}{|c|}{ Aportes ( $m \$ n)$} \\
\hline & & & & & & DNV & Consorcios Camineros \\
\hline Buenos Aires & 110 & 745 & 818 & $31.425,20$ & 302.636 .585 & 195.299 .133 & 107.337 .453 \\
\hline Catamarca & 16 & 35 & 26 & 230,3 & 28.304 .662 & 15.898 .292 & 12.406 .377 \\
\hline Córdoba & 1 & 224 & 363 & $24.360,20$ & 134.920 .929 & 98.644 .275 & 36.276 .654 \\
\hline Corrientes & 34 & 89 & 94 & $1.736,70$ & 36.071 .637 & 27.436 .793 & 8.634 .844 \\
\hline Chaco & 29 & 141 & 156 & $2.136,80$ & 72.077 .982 & 40.976 .810 & 31.101 .171 \\
\hline Chubut & 12 & 33 & 33 & $1.449,60$ & 33.722 .085 & 26.603 .035 & 7.119 .049 \\
\hline Entre Ríos & 54 & 90 & 40 & 486,40 & 54.066 .633 & 40.590 .132 & 13.476 .501 \\
\hline Formosa & 1 & 15 & 22 & 429,80 & 31.094 .943 & 24.875 .955 & 6.218 .988 \\
\hline Jujuy & 5 & 33 & 32 & 132,80 & 23.666 .989 & 17.731 .796 & 5.935 .192 \\
\hline La Pampa & 60 & 119 & 152 & $10.285,80$ & 52.534 .567 & 35.448 .781 & 17.085 .785 \\
\hline La Rioja & 19 & 22 & 12 & 147,30 & 24.100 .355 & 17.739 .649 & 6.360 .705 \\
\hline Mendoza & 1 & 76 & 77 & $2.019,30$ & 72.020 .958 & 54.564 .244 & 17.456 .714 \\
\hline Misiones & 26 & 30 & 28 & 742,40 & 26.570 .361 & 20.876 .720 & 5.693 .641 \\
\hline Neuquén & 3 & 8 & 24 & 555,80 & 18.584 .474 & 14.867 .578 & 3.716 .895 \\
\hline Río Negro & 1 & 40 & 136 & $3.380,70$ & 48.946 .731 & 35.878 .064 & 13.068 .666 \\
\hline Salta & 22 & 70 & 62 & 909,70 & 81.160 .058 & 54.842 .407 & 26.317 .651 \\
\hline Sam Juan & 16 & 116 & 75 & 517,00 & 35.613 .256 & 24.914 .578 & 10.698 .678 \\
\hline San Luis & 1 & 72 & 51 & $2.357,20$ & 26.625 .418 & 19.881 .818 & 6.743 .599 \\
\hline Santa Cruz & 8 & 15 & 17 & $1.158,30$ & 28.314 .814 & 21.296 .173 & 7.018 .641 \\
\hline Santa Fe & 282 & 338 & 487 & $13.323,60$ & 113.536 .151 & 83.598 .384 & 29.937 .767 \\
\hline Sgo. del Estero & 22 & 92 & 122 & $2.040,20$ & 58.413 .682 & 43.536 .729 & 14.876 .953 \\
\hline Tierra del Fuego & 2 & 18 & 9 & 67,30 & 2.860 .312 & 2.150 .337 & 709.975 \\
\hline Tucumán & 13 & 75 & 59 & $2.440,20$ & 77.323 .566 & 58.678 .169 & 18.645.397 \\
\hline Totales & 738 & 2496 & 2895 & $102.332,60$ & 1.383.167.148 & 976.329 .852 & 406.837 .296 \\
\hline
\end{tabular}

Fuente: Dirección Nacional de Vialidad. ${ }^{23}$

Aclaración: dentro de la longitud total se encuentra incluido el kilometraje de obras de conservación, repetido en algunos casos varias veces por sucesivas renovaciones de los convenios correspondientes. 
El éxito del Plan se tradujo en una red total estimada en $400.000 \mathrm{~km}$, de los cuales alrededor de $120.000 \mathrm{~km}$ se localizaron en la provincia de Buenos Aires. ${ }^{24}$ El sistema fue despojado de recursos en los inicios de la última dictadura militar, a partir de 1976.

\section{Derivaciones en la provincia de Buenos Aires}

Las provincias se adscribieron unánimemente a los beneficios del Plan, y la provincia de Buenos Aires no fue la excepción. En efecto, luego de haber declarado la recuperación de la autarquía de la Dirección de Vialidad de la Provincia (decreto 7823/56), el gobierno declaró el acogimiento al Plan de Caminos de Fomento Agrícola en agosto de 1956, "para dotar a su territorio de una red caminera amplia, moderna, eficiente y racional, para satisfacer las necesidades crecientes de la economía provincial" ${ }^{25}$ Esta decisión se enmarca en la defensa de la acción gubernamental a favor de la vialidad como estrategia para afianzar el agro, que se esbozó durante la intervención militar (1955-1958) y se afianzó posteriormente, durante la gestión de Alende (1958-1962). Se advertía la necesidad de crear en el interior bonaerense nuevas estructuras viales que detuvieran el éxodo hacia el Gran Buenos Aires y, por ende, la deformación del desarrollo. Una de las flagrantes responsables de dicha deformación eran las rutas nacionales pavimentadas que, diseñadas de modo radial, habían relegado a las poblaciones a la condición de tributarias del crecimiento des- proporcionado de la Capital Federal. Urgía, en consecuencia, realizar una red de rutas transversales. Asimismo, el gobierno apuntaba contra un modelo de desarrollo basado en la inequidad entre el aporte impositivo que en concepto de impuesto a la nafta hacían los usuarios bonaerenses y el aporte del gobierno nacional para la construcción y cuidado de carreteras. La preocupación vial fue expuesta por el ministro de Asuntos Agrarios Reynal O'Connor ante la Junta Consultiva bonaerense en $1956 .{ }^{26}$

Los datos a los que hemos podido acceder revelan que al año y medio de la aplicación del Plan de Caminos de Fomento Agrícola, el monto de las 430 obras ejecutadas en la provincia ascendía a \$ 30.204.107 (Mapa 1). Los guarismos del Cuadro 1 expresan que allí, en relación al total del país, se formó la mayor cantidad de consorcios camineros $(29,8 \%)$, se emprendieron la mayor cantidad de obras $(28,2 \%)$, se construyó la mayor longitud de caminos (30,7\%) y se utilizó mayor cantidad de presupuesto $(21,8 \%)$. De modo que la combinación entre los aportes de la DNV y los consorcios dio lugar a una fructífera acción. 
Mapa 1 - Monto de las obras realizadas, por zonas, en la provincia de Buenos Aires en concepto del Plan de Caminos de Fomento Agrícola, de enero de 1957 a junio de 1958

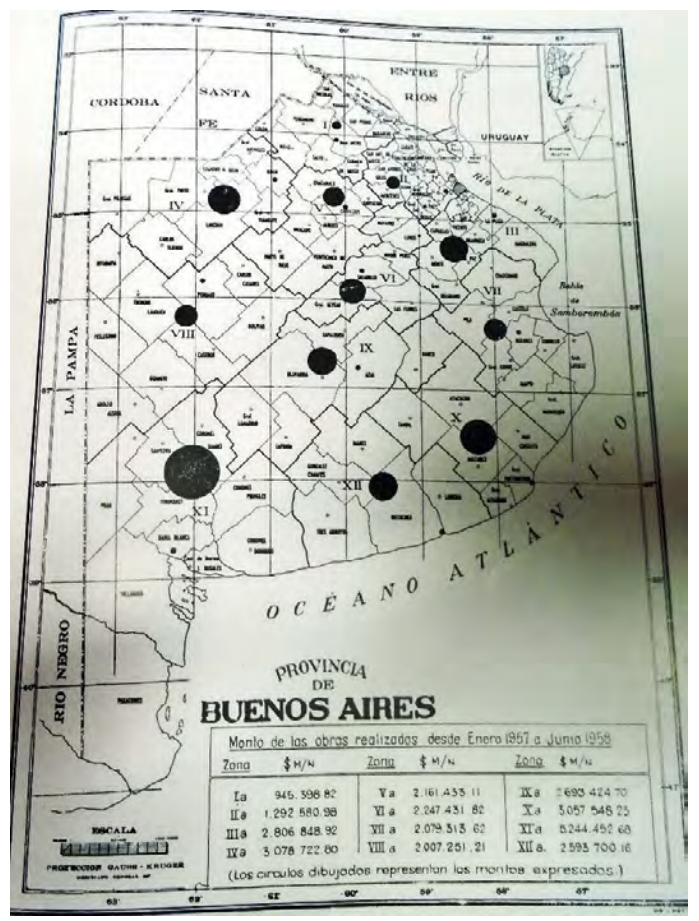

Fuente: MOP de la Provincia de Buenos Aires, Vialidad. Revista de la Dirección de Vialidad, año II, № 4, julio-agostoseptiembre 1958, p. 43.

La provincia amalgamó la obra vial con las municipalidades. En sintonía con los postulados nacionales, el gobierno provincial arengaba que la solución del problema caminero radicaba en la eficacia y la coordinación entre la nación, la provincia y las comunas, para lo cual era necesario dotar a los poderes locales de organizaciones permanentes con capacidad técnica y financiera. A esta línea de acción que apostaba por el sistema agrario y la reivindicación del ámbito local, contribuyó el régimen de Coparticipación Vial Municipal (decreto-ley 17.861/57). El mismo propendía al mejoramiento y la conservación de los caminos de jurisdicción municipal, por los que circulaba la riqueza agropecuaria, quedando excluidos aquellos localizados en zonas urbanas o urbanizadas. ${ }^{27}$ Entre los considerandos del decreto se aducía el protagonismo de la municipalidad en la tarea, la falta de recursos y la importancia de una obra vial continua, metódica y orgánica. Por este régimen, en los planes de trabajo de la Dirección de Vialidad se debía prever una partida del 10\% como mínimo total anual de recursos para distribuir entre las comunas adheridas. La distribución guardaría relación no sólo con la superficie y producción agropecuaria, sino también con la inversión municipal. De modo que, además de la extensión, se concedía prioridad a aquellas localidades que demostraran capacidad productiva y compromiso local con la obra pública (con capitales propios). Las cifras indican un notable incremento de los aportes provinciales a las comunas para la obra caminera. ${ }^{28}$ Por otro lado, vale la pena destacar la creación de doce Consejos Zonales que, integrados por los intendentes, intervenían en la aprobación de los planes de inversiones. Tenían el carácter de asesores y vinculaban a las distintas comunas.

El énfasis en los caminos municipales se reconoce también en el decreto-ley 12.186/61, por el cual la Provincia se acogió a la ley nacional 15.274/60. Indicó expresamente que la inversión de estos fondos sería para caminos vecinales no comprendidos en la red provincial primaria y secundaria. Por último, aunque amerita un análisis exhaustivo y particular, merece mención el Plan Vial 1959-1963, como vía de generación de nuevas fuentes de riqueza y de descentralización social, política 
y económica de la provincia. Tendía a estimular el desarrollo de las zonas económicamente rezagadas y permitía convenir con las municipalidades la realización de caminos de las redes provincial y comunal.

En síntesis, fue en una coyuntura marcada por las expectativas en torno a la descentralización, la eficiencia y la promoción del agro, que el Plan de Caminos de Fomento Agrícola impulsó en la provincia de Buenos Aires iniciativas viales que excedían el sistema de caminos troncales. El Estado provincial comenzó a ofrecer mayor ayuda financiera a las comunas y les imponía a cambio una serie de obligaciones: la planificación y fiscalización técnica de las obras y la contribución con fondos propios.

\section{Reflexiones finales}

La reflexión sobre la obra vial rural como política pública en la Argentina luego de la caída del peronismo no puede desligarse de un análisis de los procesos políticos, económicos, sociales e ideológicos de esa época. Aunque formaban parte de una historia vial más extensa, que halló en los años treinta un momento de consolidación, la especificidad que alcanzaron los caminos rurales a mediados de los cincuenta se vinculó con determinadas condiciones políticas, económicas e ideológicas. En el plano político, el período se caracterizó por el derrocamiento de Juan D. Perón, que inauguró recambios de dirigencias, elencos técnicos y agendas de gobierno, así como un énfasis discursivo en la democratización y la descentralización. En el plano económico, se registró un crecimiento de la actividad agraria destinada a los mercados externos, en especial durante los sesenta. Este proceso no sólo encontró un soporte en la difusión de tecnología, sino también en políticas más favorables hacia el sector. El paulatino retroceso del ferrocarril y la agudización del éxodo rural resultan telón de fondo y punto de referencia en la temática. Además, cabe destacar la influencia del desarrollismo, en el sentido de su defensa del Estado como proveedor de infraestructura para alcanzar el desarrollo. La confluencia de esta serie de cambios impulsó una acción pública más definida y sistemática sobre los caminos municipales o terciarios.

Ya desde la década de1920, en la Argentina fue cobrando fuerza una acción pública orientada al diagnóstico y a la búsqueda de soluciones en materia vial, delineándose un consenso en torno a que los caminos constituían una clave para el progreso material y moral de la nación. Ahora bien, ¿en qué sentido era nueva, entonces, la aspiración de control territorial plasmada a mediados de los años cincuenta en el Plan de Caminos de Fomento Agrícola? En primer lugar, el sistema de caminos municipales pasó a ser pensado de modo integral por el Estado, delineando un esquema de financiamiento y una estructura organizativa descentralizada, en coordinación con las direcciones de vialidad provinciales, los municipios y los vecinos. La orientación a la descentralización implicaba una transferencia de la nación a las provincias, municipalidades e incluso instituciones particulares. Junto a entidades vecinales, diversas reparticiones públicas trabajaron mancomunadamente en la tarea de cimentar una mayor apropiación del territorio nacional. La revalorización del territorio local y de sus habitantes y auto- 
ridades fue contemporánea de la obra de construcción de caminos rurales.

En segundo lugar, el sistema de caminos municipales abría la posibilidad de colaboración directa de los beneficiarios, a través de la conformación de consorcios. En rigor, si bien éstos ya detentaban status legal desde los años treinta, el Plan ofició de disparador de expectativas colectivas en torno a los mismos. La disponibilidad de recursos y la reglamentación encontraron bases en múltiples actores y fuerzas sociales movilizadas.

En suma, la novedad se expresó en el tipo de intervención, en la definición del beneficiario así como en la participación de los distintos actores. El Estado nacional, a través del Plan, marcó su presencia en la red municipal y su acción se manifestó fuertemente en las zonas rurales. Si bien el Estado se arrogaba un papel clave para el desarrollo de las acciones viales, se valoraba la participación de distintos organismos y niveles gubernamentales, así como organizaciones de la sociedad civil. Preveía de este modo una intervención coordinada -con el aporte de recursos, mano de obra, materiales, asesoramiento, control, etc.- a partir de una planificación cuyas coordenadas debían estar planteadas por la DNV. El sujeto de la intervención pública era el productor rural, necesitado de un transporte más ágil y económico que lo comunicara con los centros de consumo y lo arraigara al campo.

El Plan encontró aceptación en el gobierno de la provincia de Buenos Aires, tal como lo evidencia la cantidad de obras realizadas, la proliferación de consorcios camineros y la legislación vial complementaria. Con esta última se apuntaba a compatibili- zar tres cuestiones esenciales: el respeto al federalismo, la contribución de la comunidad local y el financiamiento. La piedra de toque era la ayuda federal y provincial, con la que se construirían caminos municipales. De esta manera, se cristalizó un consenso en torno a la cuestión caminera que excedió al sistema de caminos troncales. Si bien el tema es complejo y la evaluación puntual de su evolución e impacto supera los alcances de este trabajo, deja planteada la visibilidad que adquirió la concepción de los caminos municipales como cuestión pública.

\section{Resumo}

As estradas rurais desempenham um papel importante no desenvolvimento de atividades produtivas e na melhoria das condições de vida da população rural. Devido ao seu impacto socioeconômico e por ser uma área relativamente pouco estudada, o objetivo deste trabalho é reconstruir as políticas públicas relativas às estradas rurais argentinas, com o foco colocado sobre o Plan de Caminos de Fomento Agrícola (sancionada em 1956). Serão analisados seus antecedentes e as alterações introduzidas num contexto marcado pela modernização da agricultura nos pampas, bem como a centralidade da ideia de desenvolvimento. Da mesma forma, serão examinadas a política e as derivações institucionais no governo da província de Buenos Aires.

Palavras-chave: Argentina. Estradas. Fazenda. 


\section{Abstract}

Rural roads fulfilled an important role in the development of productive activities and the improvement of the living conditions of the rural population. Due to its socio-economic impact and that is an area relatively little studied, the purpose of the work is rebuild public policies concerning the Argentine rural roads, with focus on the Plan de Caminos de Fomento Agrícola (sanctioned in 1956). We will look at his background and changes introduced in the middle of the modernization of agriculture in the pampas and the centrality of the idea of development. We will also examine the policy and institutional derivations in the Government of the province of Buenos Aires.

Keywords: Argentina. Roads. Rural.

\section{Notas}

1 Petriz, Pedro, “La vialidad provincial en Buenos Aires", Carreteras, $\mathrm{N}^{\mathrm{o}}$ 11, julio-septiembre de 1957, p. 7.

2 Ejemplos de estos reclamos aparecen en El Tiempo (Pergamino), 29/12/1952 y 12/1/1953. El Argentino (Chascomús), 27/11/1945 y 30/8/1953.

3 Petriz, Pedro, "La vialidad provincial en Buenos Aires", Carreteras, $N^{0} 11$, julio-septiembre de 1957, p. 7.

4 República Argentina (RA), Boletín Oficial, $N^{\circ}$ 4.168, p. 86.

5 Touring Club Argentino, Memoria General del Primer Congreso Nacional de Vialidad, Buenos Aires, 1923, p. 115.

6 Touring Club Argentino, Memoria General del Primer Congreso Nacional de Vialidad, Buenos Aires, 1923, p. 115.

7 Cámara de Diputados de la Nación, Diarios de Sesiones, 23/9/1954, tomo III, p. 1817.
8 Raúl Prebisch, Informe preliminar acerca de la situación económica, Buenos Aires, 1955. Raúl Prebisch, Moneda sana o inflación incontenible. Plan de restablecimiento económico, Buenos Aires, 1956.

9 RA, Ministerio de Obras Públicas (MOP), Administración General de Vialidad Nacional, Memoria. 1954-1955-1956, p. 11.

10 Asociación Argentina de Carreteras, El camino argentino, pasado, presente y futuro, Buenos Aires, 1999 , p. 26-27.

11 RA, MOP, Administración General de Vialidad Nacional, Memoria. 1954-1955-1956, p. 15.

12 RA, MOP, Administración General de Vialidad Nacional, Memoria. 1957-1958, 1960, p. 119-120.

13 Naciones Unidas, Desarrollo de la comunidad y desarrollo nacional: informe del Grupo Especial de Expertos designado por el secretario general de las Naciones Unidas, Nueva York, 1963, p. 4.

14 MOP de la Provincia de Buenos Aires, Dirección de Vialidad, Segundo Congreso Vial de la Provincia de Buenos Aires, Publicación No 53, marzo 1965, p. 22.

15 Carreteras, $N^{\circ} 1$, enero-marzo 1955, p. 22. Carreteras, $\mathrm{N}^{\circ} 2$, abril-junio 1955, p. 40-42.

16 RA, Boletín Oficial, 8/6/1956, p.1-2.

17 RA, MOP, Administración General de Vialidad Nacional, Memoria. 1954-1955-1956, p. 37.

18 Esta idea se matiza al remontarnos a la forma de financiamiento implementada por la DNV en los años treinta, que consistía en una tasa indirecta a pagar por quien usaba el camino.

19 RA, MOP, Administración General de Vialidad Nacional, Memoria. 1954-1955-1956, p. 38.

20 Carreteras, $\mathrm{N}^{\circ} 43,1967$, p. 12.

21 La Nación, 2/6/1956, p. 2. La Prensa, 2/6/1956, p. 1. Clarín, 2/6/1956, p. 1 y 6. La Prensa, 2/6/1956, p. 1. El Argentino (La Plata), 2/6/1956, p. 1. El Día (La Plata), 2/6/1956, p. 4. Carreteras, $\mathrm{N}^{\mathrm{o}}$ 6, abril-junio de 1956, p. 2. La Cooperación, 5/10/1956, p. 1.

22 Dirección Nacional de Vialidad, extraído del Informe de la República Argentina para la segunda sesión de la Comisión Especial II del Consejo Interamericano Económico y Social: desarrollo agrícola y reforma agraria, 1963, p. 206.

23 Dirección Nacional de Vialidad, extraído del Informe de la República Argentina para la segunda sesión de la Comisión Especial II del Consejo Interamericano Económico y Social: desarrollo agrícola y reforma agraria, 1963, p. 207.

24 Carreteras, $N^{\circ} 169$, marzo de 2003, p. 15.

25 Provincia de Buenos Aires (PBA), Ministerio de Gobierno, Registro Oficial, 1956, v. VII, p. 15641565. 
26 Asuntos Agrarios, Publicación del Ministerio de Asuntos Agrarios, año IV, $\mathrm{N}^{\circ} 37$, septiembre de 1956, p. 1 y 9.

27 PBA, Ministerio de Gobierno, Registro Oficial, 1957, v. X, p. 1067-1072.

28 Por ejemplo, se preveía que de $\$ 20.000 .000$ en 1958 se pasara a $\$ 50.000 .000$ en 1960 . MOP de la Provincia de Buenos Aires, Vialidad. Revista de la Dirección de Vialidad, año II, $\mathrm{N}^{\circ} 4$, julio-agosto-septiembre 1958, p. 42.

\section{Bibliografía}

ALTAMIRANO, Carlos. Desarrollo y desarrollistas. Prismas, Buenos Aires, UNQ, n. 2, p. 75-94, 1998.

BALLENT, Anahí. Ingeniería y Estado: la red nacional de caminos y las obras públicas en la Argentina, 1930-1943. História, Ciências, Saúde - Manguinhos, Rio de Janeiro, v. 15, n. 3, p. 827-847, 2008.

Kilómetro Cero: la construcción del universo simbólico del camino en la Argentina de los años treinta. Boletín del Instituto de Historia Argentina y Americana Dr. Emilio Ravignani, Buenos Aires, n. 27, p. 107-136, 2005.

BALLENT, Anahí; GORELIK, Adrián. País urbano o país rural: la modernización territorial y su crisis. In: CATTARUZZA, Alejandro (Dir.). Nueva Historia Argentina. Tomo VII: los años treinta. Buenos Aires: Sudamericana, 2002. p. 143-200.

BARSKY, Osvaldo; GELMAN, Jorge. Historia del agro argentino. Desde la conquista hasta comienzos del siglo XXI. Buenos Aires: Sudamericana, 2001.

FERNÁNDEZ, Noelia. Políticas públicas, infraestructuras de comunicación e integración territorial en la provincia de Buenos Aires (1917-1943). In: 6 Jornada de Becarios y Tesistas, 2016, Quilmes. Anais. Quilmes: Universidade Nacional de Quilmes, 2016. p. 1-27.
FERRER, Aldo; ROUGIER, Marcelo. La economía Argentina. Desde sus orígenes hasta principios del siglo XXI. Buenos Aires: FCE, 2012.

GARCÍA HERAS, Raúl. Automotores norteamericanos, caminos y modernización urbana en la Argentina, 1918-1939. Buenos Aires: Libros de Hispanoamérica, 1985.

GERCHUNOFF, Pablo; LLACH, Lucas. El ciclo de la ilusión y el desencanto. Un siglo de políticas económicas argentinas. Buenos Aires: Planeta, 2010.

GÓMEZ, Teresita; TCHORDONKIAN, Silvia. Redes viales y ferroviarias en las décadas del treinta y del cuarenta. Documento de Trabajo, Buenos Aires, Cespa, Facultad de Ciencias Económicas, UBA, n. 40, p. 1-37, 2014.

GRUSCHETSKY, Valeria. Saberes sin fronteras: la vialidad norteamericana como modelo de la Dirección Nacional de Vialidad, 1920-1940. In: PLOTKIN, Mariano; ZIMMERMANN, Eduardo (Comp.). Los saberes del Estado. Buenos Aires: Edhasa, 2012. p. 185-211.

OSPITAL, María Silvia. Autos y caminos para la modernización de Argentina, 1920-1940. In: 18 Jornadas de Historia Económica, 2002, Mendoza. Anais. Mendoza: Editora de la Universidad Nacional de La Plata, 2002. p. 23-47.

Turismo y territorio nacional en Argentina. Actores sociales y políticas públicas, 1920-1940. EIAL, Tel Aviv, v. 16, n. 2, p. 63-84, 2005.

OSZLAK, Oscar; O’DONNELL, Guillermo. Estado y políticas estatales en América Latina: Hacia una estrategia de investigación. Documentos CEDES / Clacso, Buenos Aires, n. 4, 1981. Disponible en: <http://oscaroszlak.org. ar/articulos-esp.php>. Acesso em: 14 maio 2017.

PIGLIA, Melina. Autos, rutas y turismo: El Automóvil Club Argentino y el estado. Buenos Aires: Siglo XXI, 2014. 
PIGLIA, Melina. Gobierno provincial y clubes de automovilistas en torno a la elaboración y ejecución de la política vial en la provincia de Buenos Aires (1910-1943). In: FERRARI, Marcela; QUIROGA, Nicolás (Ed.). Historias politicas de la provincia de Buenos Aires. La Plata: Instituto Cultural de la Provincia, 2011. p. 85-114.

SCARZANELLA, Eugenia. El ocio peronista: vacaciones y 'turismo popular' en Argentina (1943 - 1955). Entrepasados - Revista de Historia 14, Buenos Aires, p. 65-84, 1998.

SKOCPOL, Theda. El Estado regresa al primer plano: estrategias de análisis en la investigación actual. In: ACUÑA, Carlos (Comp.). Lecturas sobre el Estado y las políticas públicas: retomando el debate de ayer para fortalecer el actual. Buenos Aires: Proyecto de Modernización del Estado, Jefatura de Gabinete de Ministros de la Nación, 2007 [1989]. p. 169-202.

TAMAYO SÁEZ, Manuel. El análisis de las políticas públicas. In: BAÑÓN, Rafael; CARRILLO, Ernesto (Comp.). La nueva Administración Pública. Madrid: Alianza Universidad, 1997. Disponible en: <http://politicas.typepad. com/files/tamayo-saez_-el-analisis-de-las-politicas-publicas.pdf>. Acesso em: 14 maio 2017. 